\title{
Ambientes de aula propicios para el desarrollo rítmico*
}

\author{
Javier Guerrero Jaramillo** \\ Rodrigo Andrés Romero Sánchez ${ }^{* * *}$
}

Recibido: diciembre 12 de 2015 - Evaluado: enero 18 de 2016

Aceptado: febrero 9 de 2016

Resumen

El presente artículo responde a la necesidad de hacer visibles los resultados del proyecto de investigación Ambientes que favorecen el desarrollo del sentido rítmico del estudiante de ciclo 3, realizado con la intención de analizar contextos y establecer parámetros para el diseño e implementación de un proyecto de aula exitoso. Con él se pretende garantizar el aprendizaje significativo de la música en un contexto de escuela pública: el colegio Nicolás Buenaventura. El proyecto parte de la necesidad de potenciar la habilidad rítmica dentro de la dinámica de clases formales de música, considerando el ritmo como eje formativo central.

" Investigación cualitativa de carácter descriptivo con diseño investigación - acción. Inicia en diciembre de 2014 y culmina en noviembre de 2015.

"* Licenciado en música. Aspirante a Magister en Pedagogía. Docente en propiedad Secretaría de Educación Distrital. Correo electrónico: guerremillo@gmail.com

Licenciado en música. Magister en Pedagogía. Docente en propiedad Secretaría de Educación Distrital. Asesor y jurado de trabajos de investigación de la Universidad de la Sabana. Correo electrónico: rodrialcielo@hotmail.com 
El trabajo es un estudio descriptivo que emplea instrumentos relacionales para caracterizar las situaciones que afectan a un grupo objeto de estudio, que permiten analizar los imaginarios sociales, las percepciones y formas de actuar respecto a la música. Además, persigue entender el pensamiento rítmico de los estudiantes, en función de una conceptualización acorde a su desempeño rítmico.

Palabras clave: sentido rítmico, habilidad rítmica, ambientes, unidad didáctica. 


\title{
Classroom Environments to Develop Sense of Rhythm
}

\begin{abstract}
This article answers the need to make visible the results of a research project carried out with the intention of analyzing contexts and establishing parameters for the design and implementation of a successful classroom project. The purpose of this is to ensure meaningful learning of music, in a context of a public school: Nicolás Buenaventura school. This project is based on the need to enhance the rhythmic skill within the dynamics of formal music lessons, considering rhythm as the central formative axis. The work is a descriptive study that uses correlational tools to characterize the situations involving a study group, analyzing the social imaginaries, perceptions and behaviors regarding music. In addition, it seeks to understand the rhythmic thinking of students, regarding the conceptualization according to their rhythmic performance.
\end{abstract}

Keywords: Sense of Rhythm, Rhythmic skill, Environments, Didactic unit. 


\section{Introducción}

Haciendo una revisión de investigaciones cercanas a la que origina el presente artículo, pueden considerarse, en el ámbito local, algunos estudios que han posicionado la importancia del desarrollo del sentido rítmico en la escuela, así como los que validan la influencia del ambiente en el proceso de formación musical.

Portillo (2013), con su monografía Body Percussion - Activa tu cuerpo y mente, logra la integración de estudiantes videntes e invidentes en un contexto de escuela tradicional, en respuesta a la necesidad de implementar elementos técnicos de percusión corporal como apoyos a procesos de formación musical y herramientas complementarias no sustitutivas. El investigador posiciona el ritmo como aspecto central de la música. De igual forma, Rubio (2008), en su monografía Aportes de la Taketina a la educación del sentido rítmico, señala las ventajas del método Flatischeren la educación musical y promueve su aplicación metodológica mediante talleres de exploración corporal, que fortalecen los procesos de creación e interpretación en el estudiante.

Por su parte, Miñana (1997), con Culturas musicales juveniles y escue$l a$, hace visible un eje articulador en los procesos de construcción de identidad, mediado por las culturas musicales. Analiza las necesidades de las generaciones actuales, desde las relaciones culturales entre escuela y cultura musical juvenil.

Desde una perspectiva externa, el trabajo del argentino Augusto Pérez Guarnieri (2005), plasmado en el artículo El ritmo en la educacion musical, un olvido histórico, sitúa la enseñanza del ritmo como estrategia fundamental de la construcción musical y como alternativa exitosa en la participación colectiva de los estudiantes en grupos heterogéneos de tendencias y antecedentes. Resalta la problemática del descuido del entrenamiento rítmico en los primeros ciclos y la compleja e innecesaria metodología de los ciclos más altos. El autor incita a la transversalidad de la enseñanza del ritmo en la escuela (Pérez, 2005). 


\section{Ambientes de aula propicios para el desarrollo rítmico}

En el ámbito de la educación escolar es bien sabido que aquellos niños que crecen en contextos familiares y sociales permeados por la música mostrarán ventajas en su desempeño a lo largo de su proceso de formación musical. Contar con un ambiente que expone a los estudiantes a manifestaciones musicales en la infancia, bien sea por prácticas instrumentales o hábitos de escucha y apreciación musical, es un punto a favor para el estudiante a la hora de participar activamente en los procesos de aprendizaje en el aula y al momento de asumir responsabilidad propia en su progreso.

Desde la visión de investigadores y maestros de la música como Gardner, Sloboda o Suzuki, se realza la enculturación: el aprendizaje natural que se adquiere en el ambiente al que estamos expuestos desde la infancia es uno de los aspectos determinantes en el aprendizaje de la música. Aunque es posible que se manifieste un talento "heredado", la medida con que se expresa dicho talento dependerá del medio en el que se aprenda (Gardner, 1994). Por esto, pueden considerarse a los padres como los primeros responsables en la creación de un ambiente adecuado para el aprendizaje musical de los niños.

Ahora bien, es evidente que una formación musical exitosa estará mediada por las condiciones favorables que brinde la escuela, asumiendo su papel de agente académico primario. Entonces, para lograr el ambiente adecuado para el aprendizaje musical, deben sumarse las iniciativas pedagógicas que permitan la inmersión cómoda en el estudio de la música y la aplicación del conocimiento en la práctica instrumental. La escuela será la que asuma la educación musical al considerar las premisas y las orientaciones metodológicas de los grandes maestros que han transformado la manera de enseñar la música, siguiendo la ruta trazada por académicos expertos, plasmada en lineamientos generales para la educación musical en nuestro contexto nacional.

Desde esa perspectiva, se promueve estructurar las bases para una formación de la sensibilidad, el surgimiento de la emoción musical y la tendencia por la estética desde la primera infancia y en la educación primaria. Se invita a sensibilizar a los estudiantes hacia sus motivaciones y hacia su sentido rítmico, su armonía interna, confiando en sus capacidades 
y teniendo en cuenta los contextos naturales y culturales. Se realza también, la importancia de la integración de músicas populares y la enseñanza profesional de la música, la inducción recreativa y el empleo de metodologías de entrenamiento auditivo y rítmico para lograr una capacidad perceptiva básica y una intención de inventiva musical. En relación con el proceso de transformación simbólica, se resalta la necesidad de canalizar los sentimientos, gustos e ideas de los niños a través del movimiento corporal, el manejo instrumental, la grafía y la tecnología (MEN, 2000).

Aunque la educación artística se viene posicionando en la escuela como área fundamental y obligatoria, las asignaturas de este campo siguen siendo, por lo general, relegadas a un segundo plano. En música, los enfoques más tradicionales apuntan a la historia o a la teoría, haciendo poco atractivas las clases, desconociendo el valor de la interpretación, y anulando cualquier oportunidad para la exploración corporal rítmica (Portillo, 2013). En esas condiciones, con frecuencia se incuba una "antipedagogía” de la música cuando, por un lado, se ignora la necesidad del fundamento del sentido rítmico en los primeros ciclos de educación -posible únicamente mediante didácticas de movimiento y expresión-; y por otro, se complejiza la enseñanza del ritmo en los últimos ciclos, haciendo de este algo poco llamativo y difícil de interiorizar constructivamente.

Entonces, se puede determinar que en manos de los programas académicos queda la tarea de asegurar infraestructuras educativas que apunten a la formación holística en el proceso de enseñanza musical. Para esto, han de destacarse las metodologías de educación del sentido rítmico, pensadas como una educación conjunta del movimiento, de la percepción y de la coordinación entre gesto y sonido (Martenot, 1957). La educación del ritmo puede generar una especie de conversión de los estudiantes en sujetos rítmicos, permitiendo el alivio de los defectos de orden nervioso y muscular, facilitando la libertad en su movimiento y la armonía entre sus funciones cerebrales y corporales (Centenero, 1998).

La educación musical debe servirse de todos los medios que permitan a los estudiantes comprender activa y cómodamente; y entre estos medios, el movimiento corporal es la elección más acertada. Dalcroze, en torno a la experiencia del movimiento, aseguraba que era necesario hacer pasar por el cuerpo las nociones que tradicionalmente se aprendían por medio de un aprendizaje intelectual o técnico. Por esto, insistía en la educación rítmica 
continuada y articulada a las ramas del programa escolar, resaltando la integridad del desarrollo intelectual de la mano del desarrollo físico, como la estrategia clave para lograr el desarrollo del sentido artístico (Centenero, 1998).

Por esto, y obedeciendo a la necesidad de encontrar elementos que enriquecen el proceso de articulación de planes de aula efectivos para el desarrollo del sentido rítmico, se elabora y se despliega el proyecto de investigación Ambientes que favorecen el desarrollo del sentido rítmico del estudiante de ciclo 3. El proyecto es un trabajo que busca consolidar el aprendizaje significativo de la música en contextos de escuela pública (grupos numerosos, inestables y heterogéneos), realzando la educación musical de calidad y los diseños curriculares innovadores.

$\mathrm{Al}$ iniciar el desarrollo del proyecto, se hace una medición primaria de los niveles de habilidad rítmica y de lenguajes musicales específicos en los estudiantes, y se encuentran diferencias significativas. La diversidad en las experiencias musicales, las influencias familiares y sociales, los hábitos y las tendencias que permean su cotidianidad, son los factores que se visualizan como influyentes. El interés que el estudiante muestra por su enriquecimiento académico y su formación musical es relativo a imaginarios culturales dominantes en el ambiente social o familiar, que median sus preferencias, sus actitudes de consumo hacia la música e influyen directamente en su criterio estético.

Entonces, es natural considerar tales influencias sociales como el factor que ha incidido determinantemente en las capacidades perceptivas, las intenciones expresivas y en el desarrollo motriz del estudiante de ciclo 3. El esquema concepto, transmisión y realización, expuesto por Dalcroze argumenta que un estudiante puede carecer de la noción de ritmo y del correspondiente desarrollo del sistema nervioso en relación cerebro-cuerpo y, por consiguiente, no se ha permitido el florecimiento de una clase de técnica para la expresión musical en él (Jaques-Dalcroze, 1909).

Ante la posibilidad de determinar ambientes propicios para la experiencia directa del ritmo y la interiorización de este de manera constructiva (Portillo, 2013), con el presente proyecto de investigación se busca generar propuestas didácticas desarrolladas desde la experiencia y la fundamentación académica seria, como ejes fundamentales de un proyecto de aula que ofrezca a los estudiantes mayores herramientas y garantías para 
el desarrollo sus habilidades interpretativas. Así, se da paso a la aplicación, restructuración y validación de unidades didácticas especializadas.

Considerando el sentido rítmico como elemento constitutivo de la expresión en general y eje fundamental en la motricidad y la coordinación, esta investigación puede posicionarse como un trabajo que permea y nutre distintas dimensiones del desarrollo humano: la habilidad comunicativa, la inteligencia emocional, la expresión verbal, la expresión oral, junto con las posibilidades plásticas y estéticas de los niños. En el sentido de impacto pedagógico, con la investigación se pretende entender cómo se llega a la interiorización del ritmo en el contexto escolar, estableciendo relaciones entre el desempeño rítmico y los hábitos que permean dicha interiorización. Además, la investigación busca ser una referencia para el diseño de trabajos posteriores, alusivos a la afectación del medio en el aprendizaje musical de los estudiantes, como por ejemplo el sentido estético, el consumo responsable de la música, la música como producto comercial, la percepción de los géneros musicales, la percepción de academia.

Durante el desarrollo del proyecto, se logra establecer el énfasis en el desarrollo rítmico como el modelo más efectivo en el aprendizaje musical para el contexto del colegio Nicolás Buenaventura, a partir de un estudio cualitativo descriptivo, de tipo investigación - acción. Este enfoque se percibe como una fuente de aprendizaje al alcance de todos y una ventana a la profundización en campos específicos de la música. En un contexto de escuela pública distrital, con un grupo base de estudio de estudiantes, a partir de las referencias de pares académicos, pruebas de habilidad rítmica, entrevistas y la aplicación, restructuración y evaluación de unidades didácticas, se analizan las relaciones entre las capacidades rítmicas musicales de los estudiantes y el medio al que se han expuesto y se exponen en la actualidad.

Por una parte, se puede notar que aquellos estudiantes con alto desempeño en expresión rítmica cuentan con algún tipo de experiencia o aproximación musical. Aunque son pocos los casos de parientes que cuentan con formación musical, no ha sido este un factor limitante en la adecuación de ambientes familiares que propician el desarrollo estético y la habilidad perceptiva de los estudiantes. Las prácticas instrumentales empíricas y la tendencia hacia el conocimiento y escucha de la música han favorecido el desarrollo de la habilidad rítmica en los niños. La danza y la música de baile han hecho parte de las experiencias artísticas de casa y han aportado de 
manera positiva al desarrollo de los estudiantes. En algunos casos, el fútbol ha influenciado el desarrollo motriz coordinativo de estudiantes, así como sus conductas de autorregulación y disciplina.

De otro lado, se logra determinar la necesidad y efectividad de la prueba de habilidad rítmica diagnóstica desarrollada en dinámicas libres, participativas, integradoras y cómodas: un ejercicio constructivo y propositivo que se vale de herramientas familiares para los estudiantes, que estimulan la percepción y permiten la exposición de respuestas desde las posibilidades expresivas propias. Con ésta, el estudiante logra motivarse hacia el ejercicio y permite ser evaluado consecuentemente. Así, la prueba constituye una herramienta que enriquece la práctica docente, brindando un punto de referencia importante para determinar niveles de habilidad rítmica en estudiantes de ciclo 3, en aras de propiciarles una inmersión cómoda en la materia.

Finalmente, se puede reconocer en sí misma una unidad didáctica diseñada con el modelo de Enseñanza para la Comprensión (EpC) y Aprendizaje Cooperativo, como un ambiente pedagógico que favorece el desarrollo del sentido rítmico.

Se evidencia como un hecho gratificante y constructivo en los estudiantes el contar con la oportunidad de participar en el desarrollo de talleres didácticos implementando prácticas expresivas corporales, mediante el trabajo cooperativo de pequeños grupos. Eso es así, ya que se convierte en un espacio de interacción que realza la autoconfianza y el reconocimiento por el otro, potencia la capacidad del trabajo en equipo y establece puntos de referencia desde los cuales es posible edificar el perfeccionamiento de las habilidades. En una dinámica cooperativa, pequeños grupos de trabajo procuran obtener resultados que son beneficiosos para todos sus miembros. A diferencia del aprendizaje competitivo tradicional, el cooperativo implica la evaluación continua del trabajo de los alumnos de acuerdo con criterios establecidos en conjunto (Johnson, Johnson \& Holubec, 1999). En el enfoque $\mathrm{EpC}$, los desempeños de comprensión orientan el aprendizaje de una manera organizada y visible, para realizar tareas de una manera reflexiva, retroalimentando siempre su labor, en procura de mejorar su desempeño (Perkins \& Blythe, 1994).

Con este modelo, la unidad didáctica permite potenciar el conocimiento y la habilidad rítmica, mientras se articula el "pensamiento rítmico" del estudiante. Por consiguiente, la reconstrucción conceptual y el fundamento 
del propio desarrollo del sentido rítmico se hacen posibles al traer al aprendizaje consciente los elementos que relacionan el pensamiento con el desempeño rítmico. La didáctica de la música puede ser más efectiva en la medida en que sea aplicada en pequeños grupos, permitiendo una cómoda interacción entre las partes involucradas y una clara lectura de los estilos de expresión y de exteriorización del pensamiento musical en las etapas evaluativas.

En esta situación, la dimensión emocional se convierte en un factor determinante para el buen desarrollo del trabajo. La interacción respetuosa, el autoreconocimiento y la certeza de las metas a alcanzar, inciden directamente en la apertura y en la participación del estudiante. El ambiente generado le permite captar y evaluar los estados de humor de las personas con quienes comparte el proceso pedagógico, desarrollando la habilidad para relacionarse y escanear el entorno (Díaz \& Giráldez, 2007). Así, al interactuar de manera efectiva con personas diferentes entre sí y al tomar decisiones que impliquen liderazgo en algún nivel, en alguna medida se estará potenciando la inteligencia interpersonal (Gardner, 1994). Además, dicha interacción es el escenario perfecto para emprender la comprensión de los límites propios y generar la automotivación intelectual y el autocontrol, elementos claves en la evolución de la inteligencia interpersonal (Gardner, 1994).

Con respecto a los esquemas de Enseñanza para la Comprensión, la unidad didáctica se valida como un espacio que hace tangible el aprendizaje y permite generar la retroalimentación en los procesos. Las rutinas de pensamiento permiten dicha visibilidad, coadyuvando el proceso formativo hacia un producto significativo. En el caso de unidades para un proyecto de música, la EpC permite establecer la evidencia del dominio de un tema, expuesta de manera argumentativa y propositiva (Perkins \& Blythe, 1994). Entonces, al orientar la enseñanza de una manera organizada y visible, con un diseño metodológico basado en tópicos, metas y desempeños de comprensión, adecuado para el desarrollo rítmico, se pueden consolidar las muestras musicales como una aplicación real del conocimiento, cerrando la brecha existente entre teoría y práctica, que tanto aquejan a los programas escolares de música.

En términos generales, la investigación considera los ambientes escolares, familiares y sociales como espacios en los que la música puede influir en el desarrollo de las capacidades perceptivas, auditivas de los estudiantes, así como en su interés musical. Las aproximaciones formales e informales a 
prácticas instrumentales, las intenciones formativas de los parientes, hacia la sensibilidad y los hábitos sanos de sus hijos, así como los intereses y tendencias de los círculos de amistades, son determinantes en la adquisición de elementos interpretativos, que influyen en el desarrollo del sentido rítmico de los estudiantes.

Para concluir, es preciso resaltar la importancia de las acciones investigativas y de los docentes protagonistas, comprometidos en los procesos de transformación curricular que atienden a las necesidades de los estudiantes. La práctica de aula requiere de alternativas didácticas que brinden seguridad en la enseñanza, y para esto, se hace fundamental generar ambientes que permitan el asertividad en la comunicación, la cómoda interacción personal y den paso al aprendizaje cooperativo. En un sistema educativo en el que se desconoce la expresión musical como factor de referencia en la evaluación y medición de habilidades, se hacen imperantes las propuestas pedagógicas revolucionarias que logren hacer evidente el aprendizaje significativo de la música, que rompan con los esquemas tradicionales de educación, humanizando la enseñanza hacia el despertar de la conciencia rítmica, y que permitan llevar al campo musical a posicionarse como un eje fundamental del desarrollo humano. 


\section{Referencias}

Barbosa, C. (1989). Educación Rítmica. Bogotá: Universidad Nacional de Colombia.

Centenero, M. (1998). La rítmica Jaques Dalcroze. Madrid: Pirámide.

Díaz, M. \& Giráldez, A. (2007). Aportaciones teóricas y metodológicas a la educación musical : una selección de autores relevantes. Barcelona: Graó.

Gardner, H. (1994). Estructuras de la mente, la teoría de las inteligencias múltiples. México: Fondo de Cultura Económica.

Hernández, R.; Fernández, C. \& Baptista, M. (2010). Metodología de la Investigación. Mexico D.F.: Mc Graw Hill.

Jaques-Dalcroze, E. (1909). L'éducation par le rythme. Sandoz Jobin et cie.

Johnson, D. W., Johnson, R. T. \& Holubec, E. J. (1999). El aprendizaje cooperativo en el aula. Barcelona: Paidós.

Martenot, M. (1957). Método Martenot. Buenos Aires: Ricordi.

MEN. (2000). Lineamientos curriculares para la educación artística. Bogotá: Magisterio.

Miñana, C. (1997). Culturas musicales juveniles y escuela. Bogotá: IDEP.

Pérez, A. (2005). El ritmo en la educación musical: un olvido histórico. Portal Educativo del Estado Argentino Educ.ar. Recuperado de http://portal.educ.ar/ debates/eid/musica/publicaciones/el-ritmo-en-la-educacion-musical-un-olvidohistorico.php

Perkins, D. \& Blythe, T. (1994). Putting Understanding up-front. Cambridge: Educational Leadership.

Portillo, E. (2013). Body Percussion - Activa tu Cuerpo y Mente. Tesis de grado Licenciatura en Música. Bogotá: UPN.

Rodríguez, G. (2013). Propuesta metodológica para fortalecer las habiidades rítmicas. Tesis de grado Licenciatura en Música. Bogotá: UPN.

Rubio, L. P. (2008). Aportes de la Taketina a la educación del sentido rítmico y su aplicación en la pedagogía musica. Tesis de grado Licenciatura en Música. Bogotá: UPN.

Segura Robayo, D. D., Gómez Baena, M. L. \& Lizarralde Jaramillo, M. (2007). Convivir y aprender: hacia una escuela alternativa. Bogotá: Plus medios 